\title{
Therapeutic Advances in First-Line Management of Acute Myeloid Leukemia
}

\author{
Presented by Daniel A. Pollyea, MD, MS
}

\section{ABSTRACT}

Over the past several years, drug approvals for first-line treatment of acute myeloid leukemia have changed the landscape from a "wasteland" to an "embarrassment of riches." In general, patients deemed "fit" for induction chemotherapy who have a core-binding factor abnormality should receive $7+3$ in combination with gemtuzumab, $7+3$ with midostaurin for those with an FLT3 abnormality, and liposomal cytarabine + daunorubicin for patients with secondary AML. Although other options exist, Dr. Daniel A. Pollyea recommended a venetoclax/azacitidine regimen for newly diagnosed "unfit" patients. Future research should focus on more clearly determining the definition of a "fit" patient, he said at the NCCN 2019 Annual Congress: Hematologic Malignancies.

\section{Introduction}

From the 1970s until 2017, there was little progress in the frontline treatment of acute myeloid leukemia (AML). Now, according to Daniel A. Pollyea, MD, MS, Associate Professor, University of Colorado School of Medicine, recent drug approvals in AML have led to "an embarrassment of riches" in the frontline setting (Figure 1).

Since the establishment of $7+3$ chemotherapy in the mid-1970s (7 days on standard-dose cytarabine and 3 on an anthracycline), the upfront treatment approach to AML has been decidedly binary. "We judged a patient's 'fitness' for induction chemotherapy, and if that was an available option, that is what they received," said Dr. Pollyea. "If it wasn't [an option], then they received something that didn't work very well or nothing at all."

Now, however, the landscape is quite different. At the NCCN 2019 Annual Congress: Hematologic Malignancies, he discussed the recent and burgeoning therapeutic advances in the first-line management of AML.

\section{Treatment Approach for "Fit" Newly Diagnosed Patients}

According to Dr. Pollyea, the definition of "fit" is a moving target, and deciding whether a patient is fit for induction chemotherapy continues to be a subjective, and often controversial, assessment. "But, if we agree that there are some patients who may be fit for intensive chemotherapy, then I think that [the following] is a logical assessment," he said.

In general, patients with a core-binding factor abnormality $[\mathrm{t}(8 ; 21)$ or inv $(16) / \mathrm{t}(16 ; 16)]$ should receive $7+3$ in combination with gemtuzumab. If a patient has a FLT3positive gene mutation, $7+3$ should then be paired with midostaurin. In the case of secondary AML, data support the use of a liposomal formulation of cytarabine + daunorubicin. Other patients with AML deemed fit for induction chemotherapy should be given $7+3$ alone.

\section{When Do We Use Gemtuzumab and Why?}

According to Dr. Pollyea, gemtuzumab should be used with $7+3$ chemotherapy for patients with newly diagnosed AML and good-risk disease features.

A meta-analysis of 5 randomized controlled trials of gemtuzumab in AML showed that, when stratified according to cytogenetic risk (favorable, intermediate, or adverse), patients with favorable-risk disease experienced improved outcomes, most notably those who also had core-binding factor abnormalities. ${ }^{1}$ These patients achieved a clear survival benefit with gemtuzumab, compared with a lack of survival benefit in the adverserisk group and a modest benefit in the intermediate-risk cytogenetic cohort. In these studies, gemtuzumab was incorporated into treatment on the first day. "That makes it incumbent upon us to have [cytogenetic results] at the time of treatment, since many of us regard AML as a medical emergency that is best treated yesterday," he said.

Gemtuzumab does have a potentially significant increased risk of cytopenias and liver toxicity, including vaso-occlusive disease, which is often seen in the setting of subsequent stem cell transplantation. According to Dr. Pollyea, these risks limit enthusiasm for the use of upfront gemtuzumab in patients with intermediate- and adverse-risk disease. "There is no reason to extend the toxicity of gemtuzumab to patients with adverse cytogenetic risk. They don't derive benefit and will 


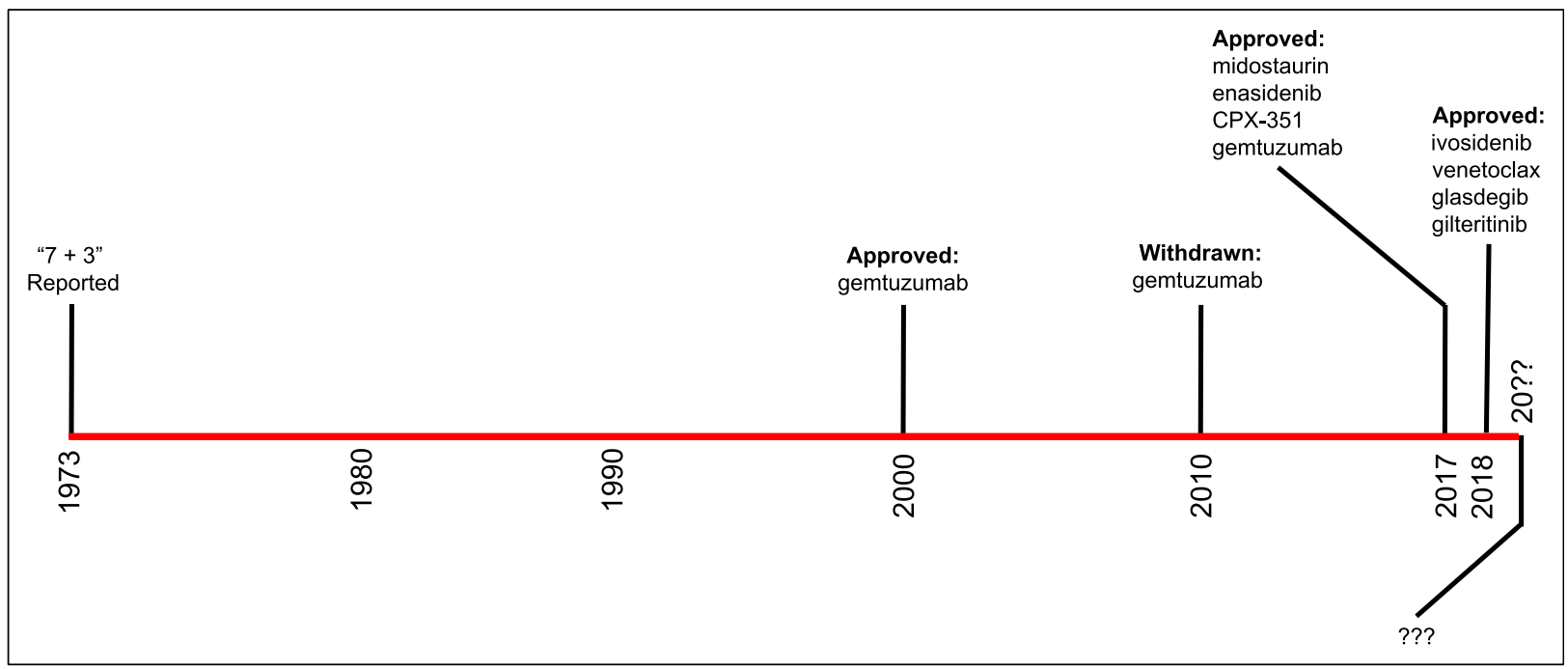

Figure 1. Recent explosion of FDA-approved drugs for use in AML.

only have the toxicity," he said. "The intermediate group, however, is a more subtle question."

\section{What About Midostaurin?}

Midostaurin (an inhibitor of FLT3) combined with $7+$ 3 has been established as the standard of care in patients with newly diagnosed AML who are fit for induction chemotherapy and have an FLT3 abnormality. This recommendation is based on data from the RATIFY study, ${ }^{2}$ which showed that patients who received this regimen experienced improved overall survival compared with those who received chemotherapy alone; this benefit was consistent across FLT3-mutated subgroups.

Dr. Pollyea noted that patients with an FLT3 abnormality typically have poor prognoses, but not because they never achieve remission - they are, in fact, responsive to treatment. Rather, the decreased overall survival rate in this population is due to recurrence of the disease.

There was also a long-term benefit of midostaurin seen in patients who went on to hematopoietic stem cell transplantation. "Transplantation is not the great equalizer that we sometimes believe it to be," he said. "The upfront treatment strategy does have an impact long term, even in the setting of a transplant."

Because midostaurin should be started on day 8 of treatment, results of FLT3 analysis should be received within the first week, he added.

\section{Liposomal Cytarabine/Daunorubicin in Secondary AML}

CPX-351, a liposomal encapsulation of cytarabine + daunorubicin, should be used in induction-eligible patients with secondary AML, which is AML that results from a known antecedent hematologic condition (eg, myelodysplastic syndromes) or a treatment-related AML. Instead of a continuous infusion over a 7-day period, CPX351 is administered intravenously over several days.

Compared with standard induction chemotherapy for newly diagnosed, induction-eligible older patients with secondary AML, treatment with CPX-351 results in a statistically significant survival benefit and superior response rates. ${ }^{3}$ Toxicities were similar to the $7+3$ regimen, except no alopecia was seen in CP-351-treated patients.

\section{Treatment Approach for "Unfit" Newly Diagnosed Patients}

"The treatment of unfit, newly diagnosed patients with AML used to be a wasteland," said Dr. Pollyea. "Up until just a few years ago, most patients were not treated at all; they were sent to hospice or received supportive care. ${ }^{4}$ Now, there are options."

$I D H 1$ and $I D H 2$ are recurrently mutated in up to $20 \%$ of patients with AML. In newly diagnosed patients with an IDH mutation, 2 single-agent IDH inhibitors have led to promising results: enasidenib for $I D H 2$ mutations and ivosidenib for $I D H 1{ }^{5,6}$ Both drugs have received FDA approval in the relapsed/refractory setting, but only ivosidenib has received an FDA label for newly diagnosed patients. "We know that there are additional benefits outside of response or survival with respect to decreased transfusion requirements in these patients, which is very promising," he noted. "And there are some emerging data showing even better responses when these therapies are paired with a hypomethylating agent."

For unfit patients without an $I D H 1$ mutation, 2 FDAapproved treatment options are available: glasdegib + low-dose cytarabine, or venetoclax + low-dose cytarabine or a hypomethylating agent. 
In unfit patients with AML or high-risk myelodysplastic syndromes, glasdegib + low-dose cytarabine demonstrated an improved survival benefit compared with low-dose cytarabine alone. ${ }^{8}$ However, Dr. Pollyea noted that response rates with glasdegib were modest, and questioned whether a median overall survival of 5 versus 9 months is clinically meaningful.

Single-agent venetoclax has led to modest response rates in patients with relapsed/refractory AML, but according to Dr. Pollyea, the "real breakthrough" came when venetoclax was paired with a backbone therapy (low-dose cytarabine or a hypomethylator) and used in patients with newly diagnosed disease. Venetoclax + low-dose cytarabine showed a $55 \%$ response rate and "very promising, durable long-term survival" for patients who achieved a response, particularly complete remission. ${ }^{9}$ However, he noted that this trial also enrolled patients with antecedent hematologic conditions who received a prior hypomethylating agent. Therefore, although all patients were not treated for AML, approximately $33 \%$ had undergone some prior therapy. When the study was adjusted for these patients, the response rate increased to nearly $70 \%$ for patients who were truly untreated for a myeloid malignancy.

In newly diagnosed, older (aged $>60$ years), unfit patients with AML who had not previously received therapy, venetoclax at $400 \mathrm{mg}$ in combination with either azacitidine or decitabine produced response rates upwards of $70 \% .{ }^{10}$ This therapy, Dr. Pollyea noted, works quickly compared with epigenetic therapies alone, with best response achieved after 1 or 2 cycles. "The expectation for response to a hypomethylating agent alone in the newly diagnosed setting is probably $20 \%$ to $30 \%$," he noted. "So, this is a meteoric rise in those numbers." Responses were similar across subsets, including cytogenetic risk, AML type, and genetic mutation; "we need more numbers to confirm this, but I don't think that this should be that surprising," he said. "All of the prognostic categories have been defined in the setting of conventional, usually induction, chemotherapy. This is a completely new regimen that works in a different way."

Although these treatment options have not been directly compared in a randomized trial, in his own practice, Dr. Pollyea opts for a venetoclax/azacitidine regimen for newly diagnosed unfit patients based on the many promising responses to venetoclax regimens.

\section{Future Questions for Fit and Unfit Patients}

According to Dr. Pollyea, future research for fit patients should focus on ways to combine specific inhibitors with $7+3$ therapy, as well as rethinking which patients should be classified as "fit" for induction chemotherapy. "For patients with adverse-risk disease, I don't think that response rates of $50 \%$ are all that promising after 45 years of the same regimen," he said. "I think it's very possible that a lot of these promising treatments we're seeing in unfit patients are going to be adapted to the fit patients, because really, who is fit for a therapy that doesn't work very well?"

Future research for unfit patients should focus on predicting lack of response to venetoclax-containing regimens, he said, as well as exploring the potential benefit of adding additional therapies to a venetoclax backbone.

Disclosures: Dr. Pollyea has disclosed that he is a scientific advisor for AbbVie, Inc., Agios, Inc., Celgene Corporation, Daiichi-Sankyo Co., Forty Seven, Inc., Janssen Pharmaceutica Products, LP, Pfizer Inc., and Takeda Pharmaceuticals North America, Inc.

Correspondence: Daniel A. Pollyea, MD, MS, University of Colorado Cancer Center, 1665 Aurora Court, Mail Stop F704, Aurora, CO 80045 Email: daniel.pollyea@ucdenver.edu

\section{References}

1. Hills RK, Castaigne S, Appelbaum F, et al. Addition of gemtuzumab ozogamicin to induction chemotherapy in adult patients with acute myeloid leukaemia: a meta-analysis of individual patient data from randomised controlled trials. Lancet Oncol 2014;15:986-996.

2. Stone RM, Mandrekar SJ, Sanford BL, et al. Midostaurin plus chemotherapy for acute myeloid leukemia with a FLT3 mutation. N Engl J Med 2017;377:454-464.

3. Lancet JE, Uy GL, Cortes JE, et al. CPX-351 (cytarabine and daunorubicin) liposome for injection versus conventional cytarabine plus daunorubicin in older patients with newly diagnosed secondary acute myeloid leukemia. J Clin Oncol 2018;36:2684-2692.

4. Medeiros BC, Satram-Hoang S, Hurst D, et al. Big data analysis of treatment patterns and outcomes among elderly acute myeloid leukemia patients in the United States. Ann Hematol 2015: 94:1127-1138.

5. Pollyea DA, Tallman MS, de Botton S, et al. Enasidenib, an inhibitor of mutant IDH2 proteins, induces durable remissions in older patients with newly diagnosed acute myeloid leukemia [published online April 9, 2019]. Leukemia. doi: 10.1038/s41375-019-0472-2

6. Westervelt P, Roboz GJ, Cortes JE, et al. Safety and clinical activity of AMV564, a CD33/CD3 T-cell engager, in patients with relapsed/refractory

acute myeloid leukemia (AML): updated results from the phase 1 first-in-human trial [abstract]. Presented at the European Hematology Association 24th Annual Meeting; June 13-16, 2019; Amsterdam, the Netherlands. Abstract S877.

7. DiNardo CD, Stein AS, Stein EM, et al. Mutant IDH1 inhibitor ivosidenib (AG-120) in combination with azacitidine for newly diagnosed acute myeloid leukemia [abstract]. Presented at 2019 SOHO Annual Meeting; September 11-14, 2019; Houston, Texas. Abstract AML-197.

8. Cortes JE, Heidel FH, Hellmann A, et al. Randomized comparison of low dose cytarabine with or without glasdegib in patients with newly diagnosed acute myeloid leukemia or high-risk myelodysplastic syndrome. Leukemia 2019;33:379-389.

9. Wei AH, Strickland SA Jr, Hou JZ, et al. Venetoclax combined with low dose cytarabine for previously untreated patients with acute myeloid leukemia: results from a phase Ib/II study. J Clin Oncol 2019;37: 1277-1284.

10. Pollyea DA, Pratz K, Jones BA, et al. Venetoclax in combination with hypomethylating agents induces rapid, deep, and durable responses in patients with AML ineligible for intensive therapy. Presented at the 60th Annual ASH Meeting; December 1-4, 2018; San Diego, California. Abstract 285. 